Please complete sample data collection forms so we can test this instrument. Thank you.

\title{
Institutional Information
}

Institution Where Patient Birthed

Brigham \& Women's

$\cap$ Mount Sinai West (Icahn School of Medicine)

$\cap$ University of Colorado, Anschutz Medical Campus (UC)

$\cap$ University of New Mexico

$\cap$ University of North Carolina

$\bigcirc$ Vanderbilt University

Equipment Type

Nitronox (Porter Instrument)

Pro-Nox (CAREstream Medical)

Encounter Number

\section{Demographics}

Date of Labor Admission

(mm-dd-yyyy)

Reason for Admission

$\Pi$ Spontaneous contraction onset

$\Pi$ Induction of labor

$\Pi$ SROM

П PROM (Rupture of membranes for the onset of contractions)

(Please check all that apply.)

Indication for Induction of Labor

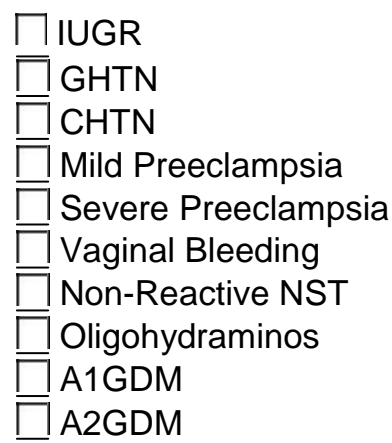

SROM

(mm-dd-yyyy hh:mm)

Maternal Age at Admission

(Age in Years)

Ethnic Category: Hispanic or Latino
Non-Hispanic
Hispanic
Refused
$\cap$ Not Available
Unknown 
Racial Category

Marital Status

Highest Degree of Education Completed

Gestational Age at Admission (Number of weeks and days in format of xx.x. Range should be xx.1 to xx.6 )

Gravidity (total \# of pregnancies regardless of duration, including current pregnancy)

Parity (\# of pregnancies carried to viable gestational age, including current pregnancy)

Prior Cesarean

Is this a singleton fetus pregnancy?

Was the fetus in a cephalic or non-cephalic presentation?

Group B streptococcus culture?

Pre-pregnancy BMI (kg/m2)

Admission BMI (kg/m2)

Total Weight Gain (in BMI)

Prescribed Medication Use Noted on Admission
American Indian or Alaska Native

Asian

Native Hawaiian or Other Pacific Islander

Black or African American

White

More than one race

O Other

O Unknown or Not Reported

Not Applicable

Single

Married

Separated

Divorced

Widowed

Elementary school (no school through completed 5th)

Middle school (some 6th through completed 8th)

Some high school (some 9th through some 12th; or working on GED)

OHigh school graduate/GED complete

ก Technical/Vocational training

Some college (includes those still in college pursuing undergraduate degree)

College graduate (includes those currently in graduate school, law school, medical school, etc.)

$\cap$ Post-graduate degree (MS, PhD, OD, CPA, etc.)

Other / Not able to be determined

$\overline{\text { (e.g., list } 38 \text { weeks and } 1 \text { day as } 38.1 \text {; range }}$ should be $x x .1$ to $x x .6$ )

(Number)

(Number)

Yes

No

Yes

No

Cephalic

Non-Cephalic

Negative

Positive

Unknown

(Number to one decimal place)

(Number to one decimal place)

(This is calculated automatically.)

$\square$ Opiate
$\square$ Benzodiazipines
$\square$ SSRIs
$\square$ Other
(Check all that apply.)


Other Prescribed Medication Use Noted on Admission:

Please list

Illicit Drug Use Noted on Admission

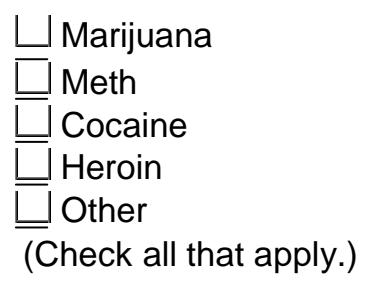

Other Illicit Drug Use Noted on Admission: Please list

\section{Prenatal Follow up}

Total Number of Prenatal Visits

(Whole number)

\section{Past Medical History}

$\begin{array}{llcc} & \text { No } & \text { Yes } & \text { Unknown } \\ \text { DM } & 0 & 0 & \bigcirc \\ \text { CHTN } & 0 & 0 & \bigcirc \\ \text { Hypothyroidism } & 0 & 0 & \bigcirc \\ \text { Hyperthyroidism } & 0 & 0 & \bigcirc \\ \text { Asthma } & 0 & 0 & \bigcirc \\ \text { Other } & 0 & 0 & \bigcirc\end{array}$

Other Past Medical History: Please list

\section{Complications in Pregnancy}

$\begin{array}{lccc} & \text { No } & \text { Yes } & \text { Unknown } \\ \text { A1GDM } & 0 & 0 & \bigcirc \\ \text { A2GDM } & 0 & \bigcirc & \bigcirc \\ \text { Mild Pre-eclampsia } & 0 & 0 & \bigcirc \\ \text { Severe Pre-eclampsia } & 0 & 0 & \bigcirc \\ \text { GHTN } & 0 & 0 & \bigcirc \\ \text { Gestational Thrombocytopenia } & 0 & \bigcirc & \bigcirc \\ \text { Preterm Labor } & 0 & \bigcirc & \bigcirc \\ \text { Other } & 0 & \bigcirc & \bigcirc\end{array}$

Other Complications in Pregnancy: Please list 


\section{Intrapartal Complications}

No

Yes

Unknown

Chorioamniotitis

Placental Abruption

Category 3 FHR Tracing

Meconium Stained Fluid

$\mathrm{PPH}$

Retained Placenta

Other

Other Intrapartal Complications: Please list

\section{Fetal Complications}

No

Yes

Unknown

IUGR

LGA

Other

Other Fetal Complications: Please list

\section{Labor and Delivery}

Onset of Labor

Was labor induced?

(mm-dd-yyyy hh:mm)

Was labor induced?

$\mathrm{O}^{\text {Yes }}$

No

Induction Method

$\sqcup$ AROM

$\sqsubseteq$ Cervical foley bulb/Cook balloon

Ð Laminaria

$\square$ Misoprostol (PGE1)

$\sqsubseteq$ Nipple stimulation / Breast Pump

1 PGE2 (e.g., Cervidil, Prepidil)

$\sqsubseteq$ Cervidil

$\sqsupseteq$ Prepidil

(Select all that apply.)

Was labor augmented?

$\bigcirc_{\text {No }}^{\text {Yes }}$

Augmentation Method

$\bigsqcup$ AROM

$\sqsubseteq$ Nipple stimulation / Breast Pump

$\sqsubseteq$ Pitocin

Ð Other

(Select all that apply.)

Other Augmentation Method: Please list 
Amniotic Fluid Character

Other Amniotic Fluid Character: Please list

Time of Complete Dilation

Date of Delivery

Fetal Position

Mode of Delivery

Type of assisted (instrumental) vaginal

Delivery Mode Indication for Operative Birth

Other Delivery Mode Indication for Operative Birth:

Please list

Perineal Lacerations

Other Perineal Lacerations

Admission Cervical Exam

Cervical Dilation (cm)

Cervical Effacement

Fetal Station

Cervical Exam Before Nitrous Initiation

How long before nitrous initiation was the exam performed?

Cervical Dilation (cm)
Clear

Meconium stained

Bloody

Purulent

Other

(mm-dd-yyyy hh:mm)

(mm-dd-yyyy)

OA

$\bigcirc \mathrm{OP}$

OT

Compound Presentation

Spontaneous vaginal

Assisted (instrumented) vaginal

Cesarean delivery

$\bigsqcup$ Forceps

$\sqsubseteq$ Vacuum

(Check all that apply.)

Fetal Intolerance of Labor

Maternal Exhaustion

Failure To Progress

Other

$\bigsqcup$ 1st degree

$\sqsubseteq$ 2nd degree

$\sqsubseteq$ 3rd degree

$\sqsubseteq$ 4th degree

$\Longrightarrow$ Sulcus

Ð Periurethral

$\amalg$ Other

(Check all that apply.)

ONo

(Number e.g., 5)

(Percentage e.g., 70)

(Number e.g., -1)

$\mathrm{O}_{\text {No }}^{\text {Yes }}$

(HH:MM (e.g., 00:20 is 20 minutes))

(Number e.g., 5) 
Cervical Effacement

Fetal Station

Cervical Exam After Nitrous Initiation

Cervical Dilation $(\mathrm{cm})$

How long after nitrous initiation was the cervical exam performed?

Cervical Effacement

Fetal Station

Placenta delivery time
(Percentage e.g., 70)

(Number e.g., -1)

$\bigcirc_{\text {No }}^{\text {Yes }}$

(Number e.g., 5)

(HH:MM (e.g., 01:00 is one hour))

(Percentage e.g., 70)

(Number e.g., -1)

(mm-dd-yyyy hh:mm)

\section{Neonate}

Infant Sex

Male

Female

Infant Birth Weight (in grams)

(Example: 2,200)

Infant Length (in inches, to one decimal place)

(Use one decimal place (e.g., 19.3))

APGAR Score: 1-minute

APGAR Score: 5-minute

APGAR Score: 10-minute (if given)

(Leave blank if $\mathrm{N} / \mathrm{A}$ )

Was neonatal resuscitation used?

$\mathrm{O}_{\text {No }}^{\text {Yes }}$

Neonatal Resuscitation

$\perp$ Warm

$\sqsubseteq$ Dry

$\sqsubseteq$ Stim PPV

$\sqsubseteq$ Chest compressions

$\sqsubseteq$ CPAP

Ð Suction

$\sqsubseteq$ Oxygen

Intubation

$\square$ Umbilical Catheter

$\square$ Neonatal Resuscitation Medications

(Check all that apply.)

Neonatal Resuscitation Medications

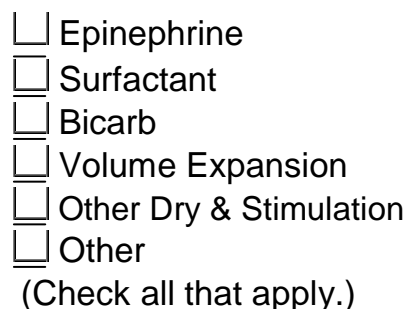


Other Medications (Please list)

Were umbilical cord gases collected?

Date and Time of Umbilical Artery Blood Collection

pHA (pH of Arterial Blood)

HCO3A (Bicarbonate) $\mathrm{mEq} / \mathrm{L}$

OSATA (O2 Saturation)

PCO2A (Partial Pressure of Carbon Dioxide in

Arterial Blood) $\mathrm{mmHg}$

PO2A (Partial Pressure of Oxygen in Arterial Blood) $\mathrm{mmHg}$

Date and Time of Umbilical Vein Blood Collection

$\mathrm{pHV}$ (pH of Venous Blood)

HCO3V (Bicarbonate) $\mathrm{mEq} / \mathrm{L}$

OSATV (O2 Saturation)

PCO2V (Partial Pressure of Carbon Dioxide in Venous Blood) $\mathrm{mmHg}$

PO2V (Partial Pressure of Oxygen in Venous Blood) $\mathrm{mmHg}$

To where was neonate originally admitted after birth?

Indication(s) for NICU (check all that apply)

Other NICU Indication(s): Please list

Duration of Hospital Stay for Infant $\bigcirc_{\text {No }}^{\text {Yes }}$

(mm-dd-yyyy hh:mm)

(Use two decimal places)

(Whole number)

(Whole number from 0-100)

(Whole number)

(Whole number)

(mm-dd-yyyy hh:mm)

(Use two decimal places)

(Whole number)

(Whole number from 0-100)

(Whole number)

(Whole number)

With Mom

NICU

Unknown

Non-viable infant

$\sqcup$ Hypoglycemia

$\sqsubseteq$ TTN

$\sqsubseteq$ Meconium Aspiration

$\sqsubseteq$ Infection

$\sqsubseteq$ Other

(Number of days, whole number) 


\section{Neonatal Feeding}

Neonatal Feeding Preference

Breast Milk Supplementation With

Indication(s) for Supplementation (check all that apply)

Other Indication(s) for Supplementation: Please list

Date and Time of Initiation of First Breastfeeding Episode
Breast Only

Breast \& Supplementation (medical indication for supplementation)

$\bigcirc$ Both Breast and Formula (patient choice is for breast and formula; not medically indicated) $\bigcirc$ Formula Only

$\Pi$ Donor Breast Milk

Formula

$\Pi$ Late Preterm Infant

$\prod$ Hypoglycemia

$\Pi$ Low Birth Weight

$\prod$ Weight Loss $>10 \%$

$\Pi$ Inadequate Milk Supply

$\Pi$ Mother Request

OOther

(dd-mm-yyyy hh:mm)

\section{Nitrous Oxide Use}

Duration of Nitrous Oxide Use (in minutes, from start to discontinuation)

Experienced $\mathrm{O} 2$ saturations <

$90 \%$ ?

Nausea during use?

Vomiting during use?

Excessive sedation during use

(sedation score 1 or 2)?

Dizziness during use?

Dry mouth during use?

Dysphoria?

Other side effects?

Other Nitrous Oxide Side Effects: Please list

Conversion to another modality?

If conversion, to which analgesia modality?

Reason for conversion to another analgesia modality?
(Whole number)

No

$\bigcirc$

0

$\bigcirc$

$\bigcirc$

O

$\bigcirc$

0

$\bigcirc$
Y Yes

No

Non-pharmacological intervention(s)

$\bigcirc$ Local/Pudendal only

ก IV/IM narcotics

$\cap$ Epidural (alone or in combination with local)

Spinal (alone or in combination with local)

Combined spinal and epidural (alone or in combination with local)

Inadequate pain relief

Side effects

Other 
Other reason for conversion to another analgesia modality: Please list

Maternal Level of Satisfaction with Nitrous Use During Administration Use a scale of 0 to 10 where 0 is Very Unsatisfied and 10 is Very Satisfied.

Pain management modalities used prior to nitrous initiation:

(Scale of 0 (Very Unsatisfied) to 10 (Very Satisfied))

$\Pi$ Non-pharmacological intervention(s)

ПLocal/Pudendal only

$\Pi$ IV/IM narcotics

$\Pi$ Epidural (alone or in combination with local)

$\Pi$ Spinal (alone or in combination with local)

$\bar{\Pi}$ Combined spinal and epidural (alone or in combination with local)

(Check all that apply.)

\section{Maternal Blood Loss and Temperature}

Estimated Blood Loss ( $\mathrm{mL})$

Uterotonic medications used in the 3rd stage:

Other uterotonic medications used in the 3rd stage:

Please list

Maternal Temperature (in degrees F) at Admission

(closest to admission time point)

Maternal Fever During Labor
(Whole number)

\begin{tabular}{l}
$\prod$ None \\
$\prod$ Pitocin \\
$\Pi$ Cytotec \\
$\Pi$ Methergine \\
$\bar{M}$ Hemabate \\
\hline Other
\end{tabular}

(Check all that apply.)

(Temperature to one decimal place)

$\bigcirc$ No, temperature never exceeded 100.4 degrees $F$ (38 degrees C)

$\bigcirc$ Yes, temperature exceeded 100.4 degrees $F$ (38 degrees $\mathrm{C}$ )

$\bigcirc$ Could not be determined (no temperatures taken in timeframe) 